\title{
In-vivo synthetic aperture flow imaging in medical ultrasound
}

\author{
Nikolov, Svetoslav; Jensen, Jørgen Arendt
}

Published in:

I E E E Transactions on Ultrasonics, Ferroelectrics and Frequency Control

Link to article, DOI:

10.1109/TUFFC.2003.1214504

Publication date:

2003

Document Version

Publisher's PDF, also known as Version of record

Link back to DTU Orbit

Citation (APA):

Nikolov, S., \& Jensen, J. A. (2003). In-vivo synthetic aperture flow imaging in medical ultrasound. I E E E Transactions on Ultrasonics, Ferroelectrics and Frequency Control, 50(7), 848-856.

https://doi.org/10.1109/TUFFC.2003.1214504

\section{General rights}

Copyright and moral rights for the publications made accessible in the public portal are retained by the authors and/or other copyright owners and it is a condition of accessing publications that users recognise and abide by the legal requirements associated with these rights.

- Users may download and print one copy of any publication from the public portal for the purpose of private study or research.

- You may not further distribute the material or use it for any profit-making activity or commercial gain

- You may freely distribute the URL identifying the publication in the public portal

If you believe that this document breaches copyright please contact us providing details, and we will remove access to the work immediately and investigate your claim. 


\title{
In-Vivo Synthetic Aperture Flow Imaging in Medical Ultrasound
}

\author{
Svetoslav Ivanov Nikolov and Jørgen Arendt Jensen, Senior Member, IEEE
}

\begin{abstract}
A new method for acquiring flow images using synthetic aperture techniques in medical ultrasound is presented. The new approach makes it possible to have a continuous acquisition of flow data throughout the whole image simultaneously, and this can significantly improve blood velocity estimation. Any type of filter can be used for discrimination between tissue and blood flow without initialization, and the number of lines used for velocity estimation is limited only by the nonstationarity of the flow. The new approach is investigated through both simulations and measurements. A flow rig is used for generating a parabolic laminar flow, and a research scanner is used for acquiring RF data from individual transducer elements. A reference profile is calculated from a mass flow meter. The parabolic velocity profile is estimated using the new approach with a relative standard deviation of $2.2 \%$ and a mean relative bias of $3.4 \%$ using 24 pulse emissions at a flow angle of 45 degrees. The 24 emissions can be used for making a full-color flow map image. An in-vivo image of flow in the carotid artery for a 29-year-old male also is presented. The full image is acquired using 24 emissions.
\end{abstract}

\section{INTRODUCTION}

$\mathrm{C}$ URRENT medical ultrasound systems acquire images by sequentially probing in the different directions of the image. Due to the relatively high speed of sound in the human tissue, this can result in a frame rate of up to 20 to 50 images a second, depending on the image size. This real-time imaging is the major advantage of ultrasound compared to X-ray, computed tomography (CT), or magnetic resonance (MR) imaging. However, the sequential acquisition does have several drawbacks. A fixed focus can be used only in transmit, and the frame rate is too low for generating high-quality, threc-dimensional (3-D), real-time images.

Attention, therefore, has turned to synthetic aperture (SA) techniques used in radar, in which a constant resolution with depth can be attained if a sufficiently large aperture array is used. Several authors have investigated this area and pointed out the problems for SA in medical ultrasound [1]-[5]. In a true SA imager, only one small element is used in transmit, and this is not sufficient to maintain the current depth of investigation and signal-tonoise ratio. However, the introduction of coded excitation and the grouping of several elements emitting a combined

Manuscript received June 10, 2002; accepted February 24, 2003 . This work was supported by grant 9700883 and 9700563 from the Danish Science Foundation and by B-K Medical A/S, Denmark.

The authors are with the Technical University of Denmark, DK $2800 \mathrm{Kgs}$. Lyngby, Denmark (e-mail: jaj@oersted.dtu.dk). spherical wave have solved this problem [4], [6]-[10]. Motion also can be a problem, because acquisition is done over several pulse emissions [1], [2], [11]-[13]. A movement of the tissue will displace the received signals and make their addition partly incoherent.

Medical ultrasound is also used for visualizing the blood flow in the human body. This is done by acquiring signals from the same image direction several times. The signals then are divided into smaller segments, and they are correlated to find either the phase or time shift from which the motion, and thereby velocity, can be estimated [14][19]. Using $N_{a}$ acquisitions for one direction reduces the frame rate by $N_{a}$, and it often becomes unacceptably low for larger flow images. The variance of the estimates are inversely proportional to $N_{a}$. To get acceptable results, a value of 8 to 16 often is needed, which is only sufficient to give qualitative flow data. Quantitative data can be acquired by imaging only in one direction, then finding the velocity distribution at a point in the vessel. But this further reduces the frame rate. A further problem is the separation between tissue and flowing blood. This has to be done over the $N_{a}$ samples from the same depth and direction. Thus, the algorithms for doing this have to work on very few samples. Due to all these problems combined, current ultrasound flow imaging is not fully quantitative, and thus gives a qualitative and angle-dependent image of the flow. Methods for solving these problems could be based on SA imaging.

The major drawback of SA imaging is, however, that the current flow imaging techniques cannot be used because the image is acquired over several pulse emissions for different transmitting sets of elements. Thus, the image is simultaneously acquired from the whole image region over several acquisitions. The data are not stationary over the acquisition time, and the individual samples cannot be directly related to one spatial position for a given time. Therefore, for flow or moving tissue, the image formed is distorted and can, in general, not be summed coherently. This has currently precluded the development of complete SA imaging systems that include velocity estimation. This paper will present a technique that solves the problem, and it is shown how the technique can be used to solve many of the problems inherent in today's sequentially acquired ultrasound velocity images.

Section II describes the basic SA image formation and how it is affected by motion. It is shown how measurements taken at different times have the same distortion if the same sequence of pulse emissions is used. Therefore, a correlation of such data can be used for finding the mo- 
tion. After every full SA image acquisition, the image data could be correlated to find the velocity. The major drawback of this is that an image is produced after a number of emissions $N$. If $N_{a}$ scan lines in the same direction is to be used for velocity estimation, the total number of emissions over which the data for the velocity estimation is collected becomes $N_{\text {tot }}=N_{a} \times N$. If $N=8$, and $N_{a}=16$, the total number of emissions exceeds 100 , providing only 16 samples over which to estimate the velocity. A way to reduce the acquisition time is to use recursive ultrasound imaging [13]. A new image then is created after every emission, and the velocity can be found continuously by correlating recursive images acquired from the same combination of emissions. This solves the long acquisition time problem.

The performance of the method is demonstrated in Section III, in which a parabolic velocity profile is simulated. Results also are shown for a measured parabolic profile in a flow rig obtained with an experimental ultrasound scanner capable of acquiring real-time, synthetic aperture data. The scanner is also used for acquiring the in-vivo synthetic aperture flow image in Section IV of the carotid artery of a healthy volunteer. The paper is summarized in Section V.

\section{IMAGING APPROACH}

Synthetic aperture images are acquired by emitting with individual elements of the aperture sequentially as shown in Fig. 1. Data are then measured for all transducer elements, and a low-resolution image can be generated by focusing the data in all directions of the image.

The focusing is done for every point in the image by coherently summing the signals received by the transducer elements. The distance from the transmitting element to the current imaging point and back to the receiving element is:

$$
d_{i j}\left(\vec{x}_{p}\right)=\left|\vec{x}_{p}-\vec{x}_{i}\right|+\left|\vec{x}_{j}-\vec{x}_{p}\right|,
$$

where $\vec{x}_{i}=\left(x_{i}, y_{i}, z_{i}\right)$ is the position of the emitting element $i, \vec{x}_{p}=\left(x_{p}, y_{p}, z_{p}\right)$ is the position of the point, and $\vec{x}_{j}=\left(x_{j}, y_{j}, z_{j}\right)$ is the position of the receiving element $j$. The propagation time from the start of the current emission is $t_{i j}\left(\vec{x}_{p}\right)=\frac{d_{i j}\left(\vec{x}_{p}\right)}{c}$, where $c$ is the speed of sound.

The focused signal is then given by:

$$
L_{i}\left(\vec{x}_{p}\right)=\sum_{j=1}^{N_{\mathrm{rev}}} s_{i j}\left(t_{i j}\left(\vec{x}_{p}\right)\right),
$$

where $s_{i j}(t)$ is the received signal for element $j$ when emitting with element $i$, and $N_{\mathrm{rcv}}$ is the number of receiving elements. The number of transducer elements is $N_{\mathrm{xdc}}$, and $1 \leq N_{\text {rcv }} \leq N_{\text {xdc }}$. The beamformed signal in a single point of the low resolution image is given in (2). The points $\vec{x}_{p}$ can be chosen randomly. Usually they are placed along a line, which is characterized by a direction and origin as shown in Fig. 2. The distance between two adjacent points along that line usually is constant. It must be noted that
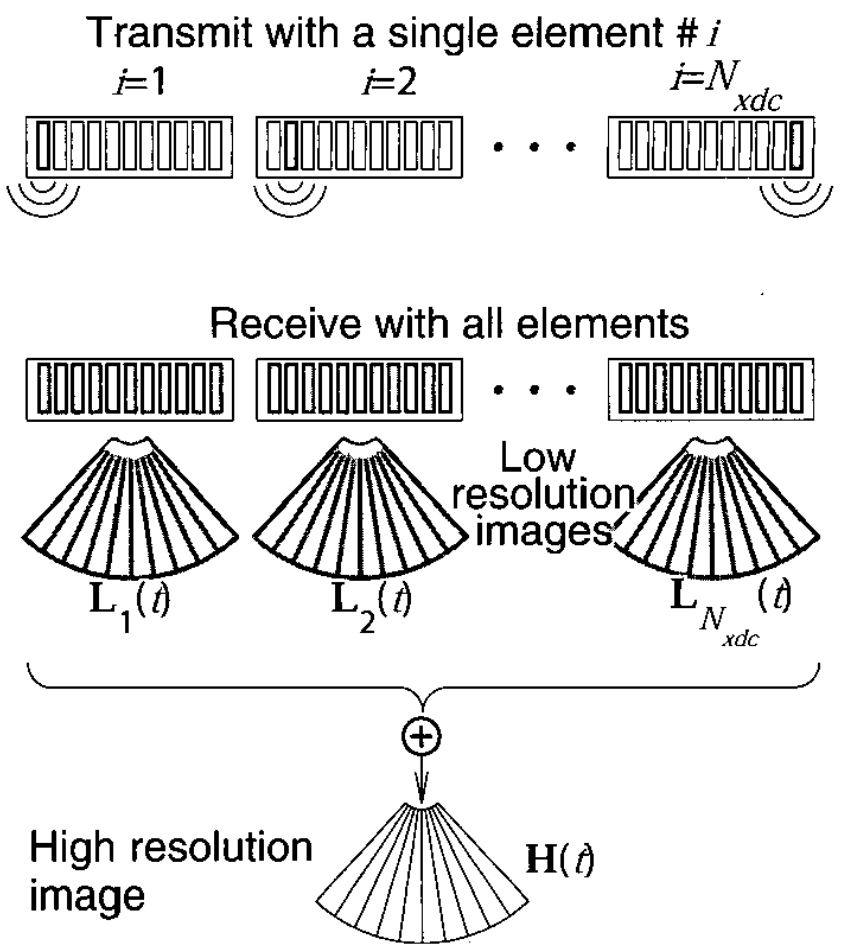

Fig. 1. Synthetic transmit aperture imaging. A number of low resolution images are created by focusing the received signal by transmitting with a single element and receiving with all elements. All the low-resolution images are then combined to form the final highresolution image at the bottom.

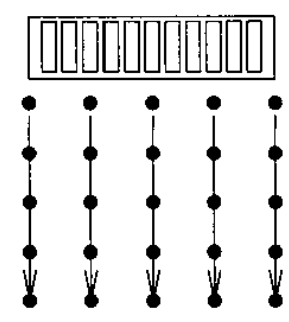

(a)

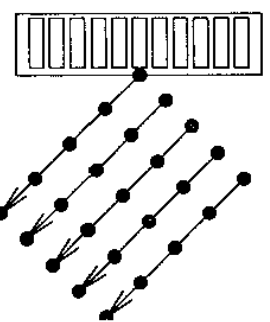

(c)
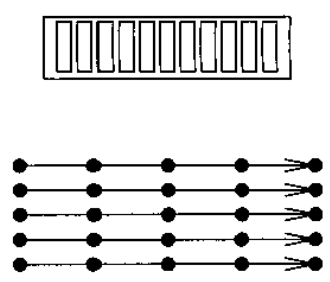

(b)
Fig. 2. SA imaging makes it possible to beamform scan lines defined by an origin and a direction. The arrows on the drawings symbolize the beamformation direction. Subfigures (a) and (d) show standard linear and phased-array images, respectively. The scan lines in (b) are parallel to the transducer surface, and the scan lines in (c) are tilted at 45 degrees to the transducer surface. 
the origin of the line, its direction, and the distance between two points can be selected arbitrary. The only requirement is to have a backscattered signal coming from these imaging points. The motion can be estimated along any of these imaging lines.

For the sake of simplicity, only phased-array images are considered in the rest of the article. The points are placed along scan lines $L_{i l}\left(\frac{c t}{2} \cdot \vec{\xi}_{l}\right)$, where $l$ is the index of the scan line, $\vec{\xi}_{l}=\left(\cos \gamma_{l}, 0, \sin \gamma_{l}\right)$ is the scan direction, and $\gamma_{l}$ is the angle between the scan line and the central line of the image as shown in Fig. 2. A low resolution, image (LRI) with $N_{l}$ scan lines can be expressed as a matrix:

$\mathbf{L}_{i}(t)=\left[L_{i 1 .}\left(\frac{c t}{2} \vec{\xi}_{1}\right), L_{i 2}\left(\frac{c t}{2} \vec{\xi}_{2}\right), \ldots, L_{i N_{l}}\left(\frac{c t}{2} \vec{\xi}_{N_{l}}\right)\right]$

Each column of the matrix $\mathbf{L}_{i}(t)$ corresponds to a separate imaging direction. The index $i$ indicates that the image was acquired after a transmission with the element $i$.

Such images are focused only in receive, and a fully focused image can be formed by emitting with different elements then adding the resulting low resolution images coherently to form a high resolution image. This is done by:

$$
\mathbf{H}(t)=\sum_{i=1}^{N} \mathbf{L}_{i}(t)
$$

where $2 \leq N \leq N_{\mathrm{xdc}}$ is the number of transmit events.

Fig. 3 shows the creation of several successive highresolution images, when a single point scatterer moves toward the transducer. The top row illustrates the emission sequence. Only the outermost two elements are used in transmit $(N=2)$, and the active one has a dark gray color. They emit sequentially: first element number one, then element number $N_{\mathrm{xdc}}$, then element number one again, and so on. $N_{\mathrm{xd} c}$ is the total number of transducer elements. The emissions have a global index $n$ and are counted from the start of the process. All of the transducer elements are used upon reception; and after every emission $n$, a low-resolution image (LRI) $\mathbf{L}^{(n)}(t)$ is formed. In (2), the index of the low-resolution images denoted the element with which the transmission was performed. If the point in Fig. 3 was stationary (no motion), then the following equations would have been true: $\mathbf{L}^{(1)}(t)=\mathbf{L}_{1}(t)$, $\mathbf{L}^{(2)}(t)=\mathbf{L}_{N_{\text {xó }}}(t), \mathbf{L}^{(3)}(t)=\mathbf{L}_{1}(t) \ldots$ The point spread functions for the LRIs are tilted at different angles to the transducer surface $[10]$. The angles depend on the position of the transrnitting element $i$.

The middle row of Fig. 3 illustrates the low-resolution images of a single point. The point moves from one emission to the next toward the transducer a distance $\Delta z$ given by:

$$
\Delta z=|\vec{v}| \cos (\theta) T_{\mathrm{prf}}=v_{z} T_{\mathrm{prf}},
$$

where $\vec{v}$ is the vectorial velocity of the moving object, $\theta$ is the angle between the ultrasound beam direction and the velocity vector, and $T_{\mathrm{prf}}$ is the time between pulse

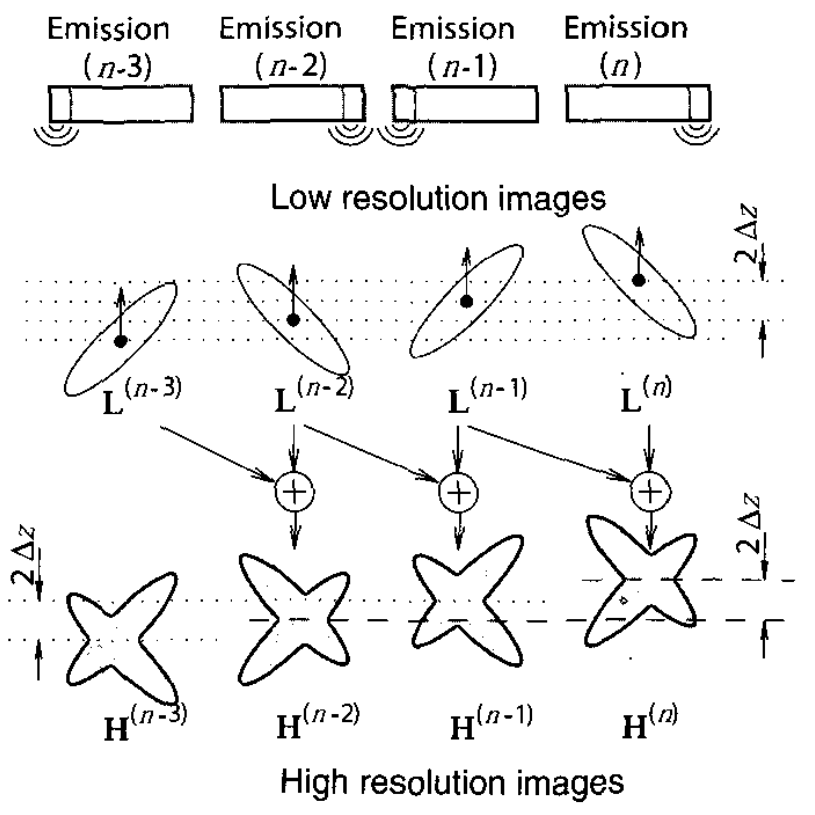

Fig. 3. Creation of several successive high-resolution images from a sequential acquisition of low-resolution images. The high-resolution images will have different point spread functions, shown at the bottom, due to the motion of the point scatterer toward the transducer.

emissions. ${ }^{1}$ It can be shown that $\mathbf{L}^{(n)}(t)$ and $\mathbf{L}^{(n-N)}(t)$ are translated versions of each other, when $\Delta z$ is small compared to the distance to the transducer [10].

At every emission, a high-resolution image can be formed by summing the last $N$ low-resolution images $[13],[20],[21]$ :

$$
\mathbf{H}^{(n)}(t)=\sum_{i=n-N+1}^{n} \mathbf{L}^{(i)}(t),
$$

where $t$ is the time from the start of the emission. If the tissue is motionless, then $\mathbf{L}^{(i)}(t) \equiv \mathbf{L}^{(i-N)}(t)$ and $\mathbf{H}^{(n)}(t) \equiv \mathbf{H}^{(n-N)}(t)$. In the presence of motion, however, $\mathbf{L}^{(n)}(t)=\mathbf{L}^{(n-N)}(t-2(N \Delta z) / c)$, where $c$ is the speed of sound. For a constant velocity, the high-resolution images, therefore, are related by:

$$
\mathbf{H}^{(n)}(t)=\mathbf{H}^{(n-N)}\left(t-2 \frac{v_{z}}{c} N T_{\mathrm{prf}}\right)
$$

where $v_{z}$ is the component of the velocity along the beam direction. This situation is depicted in the bollom row of Fig. 3. It can be seen that two successive, high-resolution images differ due to the different transmit scquences with which they were obtained; but, the high resolution images, that are $N$ transmissions apart, are shifted versions of one another.

The acquired high-resolution data for every $N^{\prime}$ th emission have the same correlation properties as conventional ultrasound flow data, and all the different methods for velocity estimation can be applied [19]. The only difference

\footnotetext{
${ }^{1}$ For the case depicted in Fig. $3, \theta=0$, and $v_{z}=|\vec{v}|$.
} 


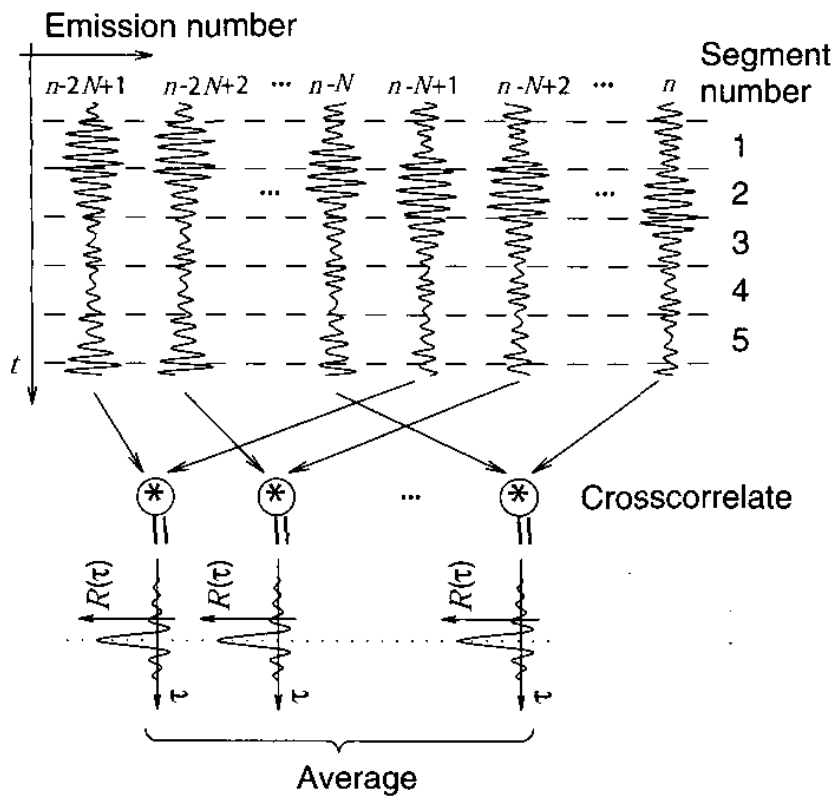

Fig. 4. Estimation of the cross-correlation function of several consecutive high-resolution lines formed in the same direction. Only lines generated from the same sequence of emissions are correlated, then all the cross-correlation functions can be averaged.

is that the pulse repetition period for the individual data sets is $N T_{\text {prf }}$ rather than $T_{\text {prf }}$. The data will be highly correlated only between $H^{(n)}(t)$ and $H^{(n-N)}(t)$ for any $n$. Therefore, the complete set of measurements can be viewed as $N$ sets of measurements with the same correlation properties within the sets.

This shift can be estimated using cross correlation of the high-resolution lines $H^{(n)}(t)$ and $H^{(n-\dot{N})}(t)$, which are formed in the same direction of the image (see Fig. 4). The cross-correlation for one pair of high-resolution lines within a segment from $t_{1}$ to $t_{2}$ is:

$$
\begin{aligned}
R_{n-N, n}(\tau) & =\frac{1}{t_{2}-t_{1}} \int_{t_{1}}^{t_{2}} H^{(n-N)}(t) H^{(n)}(t+\tau) d t \\
& =\frac{1}{t_{2}-t_{1}} \int_{t_{1}}^{t_{2}} H^{(n-N)}(t) H^{(n-N)}\left(t-t_{s}+\tau\right) d t \\
& =R_{n-N, n-N}\left(\tau-t_{s}\right)
\end{aligned}
$$

where $t_{s}=N \frac{2 v_{z}}{c} T_{\text {prf }}$ is the time shift due to motion. The peak of the cross-correlation function is located at $\tau=t_{s}$, and the velocity can be found from the time shift:

$$
v_{z}=\frac{c}{2} \frac{t_{s}}{N T_{\mathrm{prf}}}
$$

The lines at emissions $n-i$ and $n-N-i$ are subject to the same time shift and the peak of their cross-correlation function is also located at lag $\tau=t_{s}$, as shown in Fig. 4 . The different cross-correlation functions, thus, can be averaged to obtain a more precise correlation function.

In the case of a flow profile, such as the parabolic flow profile, the velocity of the blood flow changes as a function of space and time. To estimate the velocity at the different spatial positions, each of the RF lines is divided into a number of segments as shown in Fig. 4. A cross-correlation function is estimated for each of the segments. Normally the signals are digital, and the discrete version of the cross correlation is:

$$
\hat{R}_{n-N, n}\left[\eta, i_{\text {seg }}\right]=\frac{1}{N} \sum_{k=\left(i_{\text {seg }}-1\right) N_{s}}^{i_{\text {seg }} N_{s}-1} H^{(n-N)}[k] H^{(n)}[k+\eta],
$$

where $N_{s}$ is the number of samples in a segment and $i_{\text {seg }}$ is the number of the segment. The estimated velocity is:

$$
\hat{v}_{z}=\frac{c}{2} \frac{\hat{\eta}_{m} f_{\mathrm{prf}}}{N f_{s}}
$$

when the maximum occurs at $\hat{\eta}_{m}$.

The estimate can be improved by averaging the estimated cross-correlation functions:

$$
\hat{R}_{a v}^{(n)}\left[\eta, i_{\mathrm{seg}}\right]=\frac{1}{N_{c}} \sum_{l=-N_{c}+1}^{0} \hat{R}_{n-l-N, n-l}\left[\eta, i_{\mathrm{seg}}\right]
$$

if the velocity does not change significantly for several sequential acquisitions $N_{c}$. Fig. 4 shows the case for which $N_{c}=N$. If the images were created after every $N$ emissions, then the total number of emissions needed for the velocity estimate would be $N_{c} N$. For $N=8$ and $N_{c}=16$, the total number of emissions would be $N_{c} N=128$. The correlation between the high-resolution lines would decrease due to acceleration, velocity gradients, and migration of scatterers [10], [18], [19]. In the new approach, only $N+N_{c}=24$ emissions are necessary, thus preserving the high correlation between the images and giving the possibility of estimating the velocity with a low bias and variance. The first $N$ emissions are used to create the first image in the sequence. Once the process is started, there is a new image after every emission. Hence, $N_{c}$ more emissions are required to create $N_{c}$ pairs of images that are cross correlated.

It should be noted here that continuous imaging is possible. Data is always available for velocity estimation for all depths and imaging directions because the whole image region is probed simultaneously. Thus, it is not necessary to change the acquisition for probing in a different part of the image. Thus, the amount of data available is limited only by the flow physics, i.e., over how long a time it can be considered stationary with the same velocity.

The continuous availability of data is of considerable importance for stationary echo canceling. Traditionally short filters have to be used because only 8 to 16 samples are available. Now any kind of filter can be used because there are no initialization effects for continuous data. The echo canceling must be done using $H^{(n)}(t)$ and $H^{(n-N)}(t)$ and so forth. But then any high-pass filter can be selected, and lower blood velocities can be estimated. Continuous data also opens up the possibility of using adaptive filters. 


\section{RESULTS}

The performance of the new SA velocity estimation approach was investigated for a laminar, parabolic velocity profile in a tube with simulations and measurements on a flow phantom. The same setup was used for both simulations and measurements.

\section{A. Choice of Parameters}

1. Number of Transmissions: Gammelmark and Jensen [22] have shown in-vivo that synthetic transmit aperture can produce images superior to the conventional Bmode, linear-array images in terms resolution and dynamic range. Hazard and Lockwood [23] have shown for synthetic transmit aperture (STA) imaging with three emission and speeds of up to $80 \mathrm{~cm} / \mathrm{s}$ that the image resolution is preserved, the main effect of axial motion is only a slight translation of the peak of the main lobe, and a $4 \mathrm{~dB}$ increase in the level of side lobes. Therefore, the number of transmissions was limited to $N=4$.

2. Transmitted Pulse: Foster et al. [18] have shown in their error analysis that the precision of the crosscorrelation method is proportional to the bandwidth of the system and the signal-to-noise ratio. The use of a linear frequency modulated pulse increases the signal-to-noise ratio while maintaining the bandwidth and axial resolution of the system. To maintain low-range lobes, a weighting on the pulse and optimization of the compression filter is done as described by Misaridis and Jensen [24].

3. Segment Size: A longer segment improves the estimate of the cross-correlation function. However, a longer segment contains echoes from scatterers with different velocities due to spatial velocity gradients. Foster et al. [18] show that, for larger vessels, an appropriate correlation interval is between 2 and $6 \lambda$.

4. Number of Cross Correlations: The estimation procedure presented in Section II assumes stationary flow. To improve the estimate of the cross-correlation function, a number of estimates $\hat{R}\left[\eta, i_{\text {seg }}\right]$ are averaged as shown in (12). This averaging will work only if the cross-correlation functions have the same position of the maximum peak. This will be the case for stationary flow; whereas, for an accelerated flow, a gradual shift in the position of the peak will occur due to changing velocity. Such a gradual shift should be limited to no more than half a sample over the entire time cluration of the averaging. The half sample shift $\left(\Delta \eta_{m}=1 / 2\right)$ corresponds to a velocity change of:

$$
\Delta v_{1 / 2}=\frac{c}{2} \frac{\frac{1}{2} f_{\mathrm{prf}}}{N \cdot f_{s}} .
$$

This must be less than the acceleration of the flow times the averaging time, and hereby:

$$
a \times T_{\text {meas }}=a \frac{N_{c}}{f_{\mathrm{prf}}}<\frac{c}{4} \frac{f_{\mathrm{prf}}}{N \cdot f_{s}} .
$$

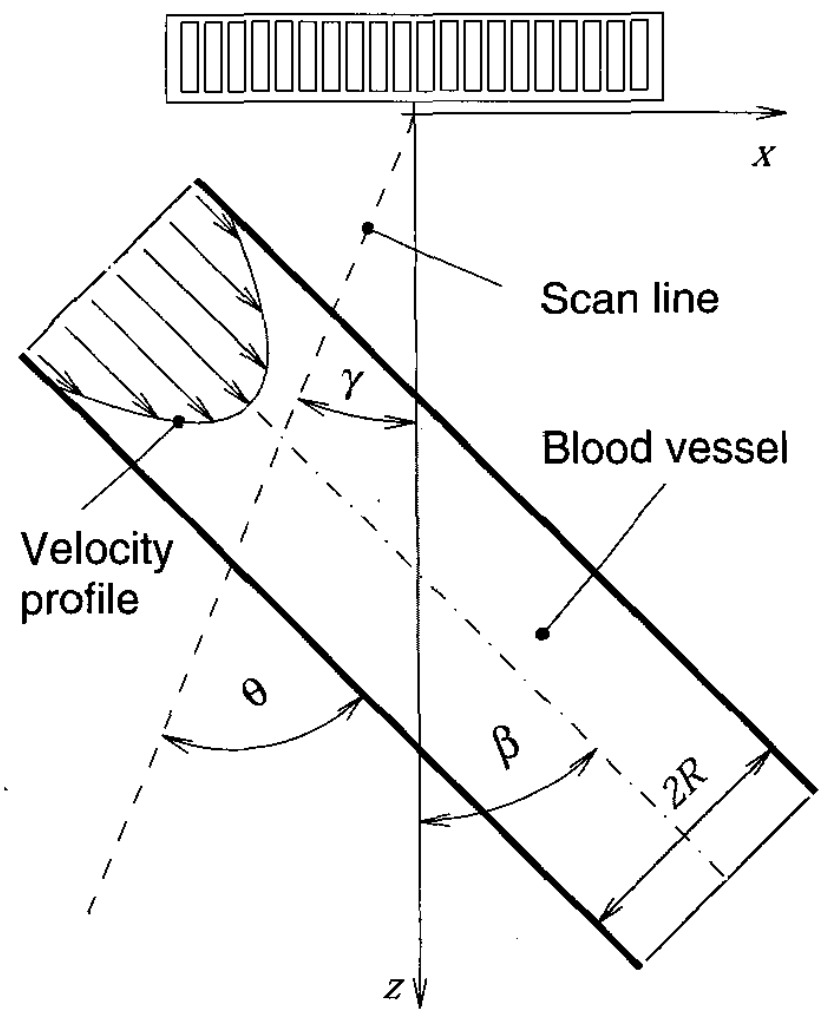

Fig. 5. Simulation setup for a flow with parabolic velocity profile in a circular tube.

Dilley and Fronek [25] report a peak acceleration for the femoral artery of up to $3.53 \pm 1.12 \mathrm{~m} / \mathrm{s}^{2}$. In a typical imaging situation, a pulse repetition frequency of $f_{\mathrm{prf}}=$ $7000 \mathrm{~Hz}$ will allow imaging of up to $10 \mathrm{~cm}$ in depth. Setting $N=4, f_{s}=40 \mathrm{MHz}, c=1540 \mathrm{~m} / \mathrm{s}$, and $a=5 \mathrm{~m} / \mathrm{s}$, one gets a maximum averaging time of $T_{\text {meas }} \approx 3.3 \mathrm{~ms}$. The latter figure means that, for a given segment, the peak of the cross correlation will not shift with more than half a sample for at least $N_{c} \approx 24$ pulse-echo events for this set of parameters.

\section{B. Simulations}

The simulations were done using the program Field II [26]. The setup is-shown in Fig. 5; the angle between the scan line and the center image line is $\gamma, \beta$ is the angle between the flow direction and the center image line, and $\theta$ is the angle between the flow and the image line along which the flow is being estimated. The radius of the vessel is $R=5 \mathrm{~mm}$, and the length of the vessel is $50 \mathrm{~mm}$.

The number of point scatterers simulated is $N_{\text {scat }}=$ 78,549 . The maximum velocity at the center of the vessel is set to $\left|\vec{v}_{\max }\right|=0.178 \mathrm{~m} / \mathrm{s}$ because this is the velocity obtainable with the measurement setup in Section III-C. The velocity profile is parabolic, and it is shown with arrows in Fig. 5 and is given by:

$$
\vec{v}(r)=\vec{v}_{\max }\left(1-\left(\frac{r}{R}\right)^{2}\right), \quad|r| \leq R,
$$


TABLE I

Measurement Parameters for the Simulations, the Flow Rig, And IN-Vivo Measurements.

\begin{tabular}{lcc}
\hline Parameter & Value & Unit \\
\hline Transducer center frequency & 7.5 & $\mathrm{MHz}$ \\
Relative transducer bandwidth & 66 & $\%$ \\
Number of elements & 64 & - \\
Transducer pitch & 205 & $\mu \mathrm{m}$ \\
Transducer kerf & 30 & $\mu \mathrm{m}$ \\
Transducer height & 4 & $\mathrm{~mm}$ \\
Elevation focus & 25 & $\mathrm{~mm}$ \\
Excitation type & chirp & - \\
Excitation start frequency & 0 & $\mathrm{MHz}$ \\
Excitation end frequency & 17 & $\mathrm{MHz}$ \\
Excitation duration & 20 & $\mu \mathrm{s}$ \\
Sampling frequency & 40 & $\mathrm{MHz}$ \\
Pulse repetition frequency & 7000 & $\mathrm{~Hz}$ \\
Speed of sound & 1485 & $\mathrm{~m} / \mathrm{s}$ \\
\hline
\end{tabular}

where $r$ is the radial position in the vessel. The angle between the vessel and the normal vector to the transducer surface is $\beta=45^{\circ}$, and the center of the vessel is placed $50 \mathrm{~mm}$ from the transducer surface. The velocity was estimated for different angles $\theta$ between the ultrasound beam and the vessel.

The simulation parameters are given in Table I. A 64elements transducer with a nominal frequency of $7.5 \mathrm{MHz}$ was simulated. The excitation was a linear frequency modulated (FM) signal as described in [24] to simulate a real synthetic aperture imaging system, which uses codes to increase the signal-to-noise ratio [10]. The compression filter was applied before the velocity estimation. The number of elements used in transmit is $N=4$ equally spaced across the aperture. The results for the central line in the image $\left(\gamma=0^{\circ}, \beta=45^{\circ}, \theta=45^{\circ}\right)$ are given in Fig. 6. The black dashed line is the true velocity profile. The black solid line is the mean estimated velocity, and the gray solid lines outline the \pm 3 standard deviations of the estimated velocity profile. Fig. 6(a) is obtained by averaging over $N_{c}=24$ cross-correlation functions $R_{n-4, n}(\tau)$. The correlation interval was $2.5 \lambda$ long, and the search range was limited to $\pm 1.7 \lambda$. The velocity was estimated at every $0.5 \mathrm{~mm}$ along the scan line, and the estimates were compensated for the $\cos \theta$ factor in (5). The mean velocity profile and the standard deviations were calculated from 189 velocity profiles. The maximum bias of the estimated profile is $-3.05 \%$, and the maximum standard deviation is $2.7 \%$. Averaging over only $N_{c}=4$ cross correlations gives the profile shown in Fig. 6(b). In order to maintain the low standard deviation, the length of the correlation interval was increased to $5 \lambda$. The interval in which the maximum of the correlation function is sought was increased to $\pm 2.5 \lambda$. The standard deviation relative to the peak velocity of $0.178 \mathrm{~m} / \mathrm{s}$ varies from $1.2 \%$ at the center of the vessel $(50 \mathrm{~mm}$ from the transducer surface) to $2.5 \%$ at the boundary of the vessel (42 $\mathrm{mm}$ from the transducer surface). The bias is maintained below $3.5 \%$.

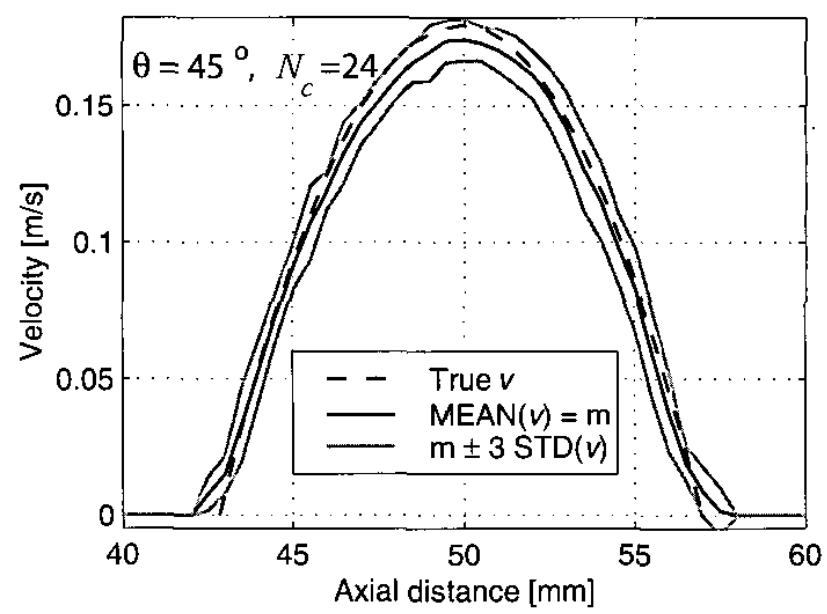

(a)

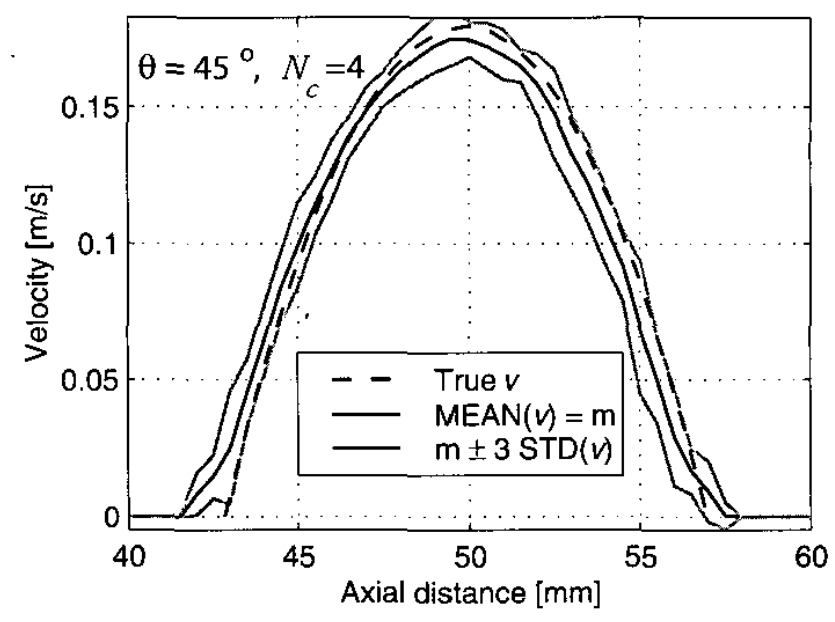

(b)

Fig. 6. Averaged simulated velocity profile for $\theta=45^{\circ}$. The estimates were obtained by averaging over (a) $N_{\mathrm{c}}=24$, and (b) $N_{c}=4$ correlation functions.

Using the same set of measured data, a whole image of the blood flow can be created. Fig. 7(a) and (b) show the velocity profiles for $\theta=35^{\circ}$ and $55^{\circ}$, respectively. The number of cross correlations over which the estimate was averaged was 4 . The standard deviation and bias are on the same order as for $\theta=45^{\circ}$, about $3 \%$.

\section{Phantom Measurements}

The performance of the new SA velocity estimation approach was further investigated for a laminar, parabolic velocity profile in a tube. The measurement setup is shown in Fig. 8. The flow rig consists of a Smedegaard Type EcoWatt 1 pump (T. Smedegaard A/S, Glostrop, Denmark), and the mean flow is measured by a MAG 1100 mass flow meter (Danfoss A/S, Nordborg, Denmark) by Danfoss. The length and diameter of the tube are $1 \mathrm{~m}$ and $17 \mathrm{~mm}$, respectively, to ensure a fully developed parabolic and laminar flow. The true velocity profile is derived from the mass flow meter and the geometric tube dimensions. A blood 


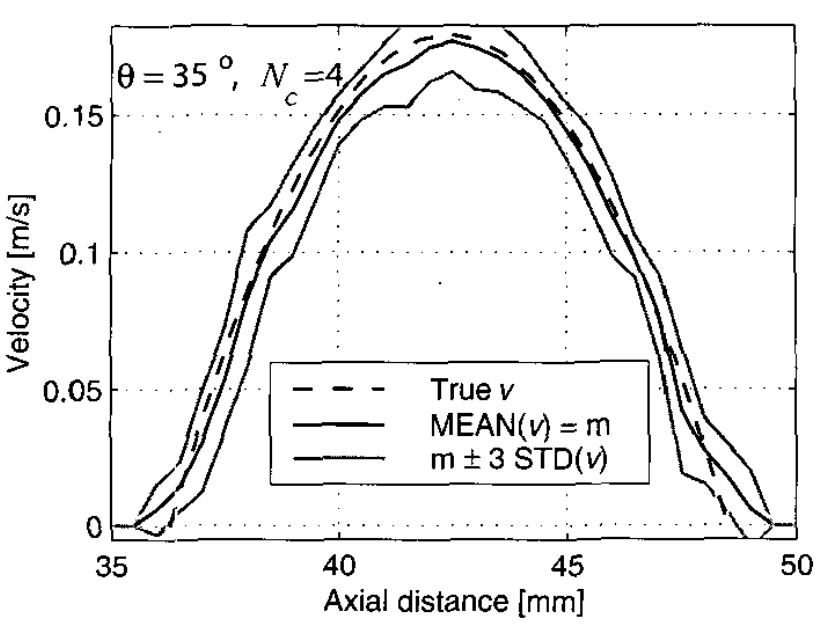

(a)

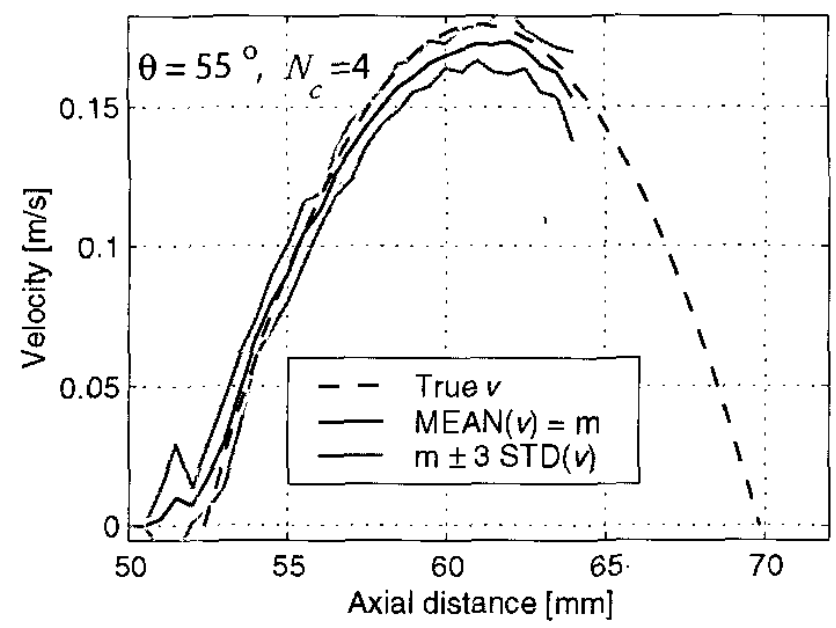

(b)

Fig. 7. Velocity profile obtained by averaging over $N_{C}=4$ crosscorrelation functions for (a) $\theta=35^{\circ}$, and (b) $\theta=55^{\circ}$.

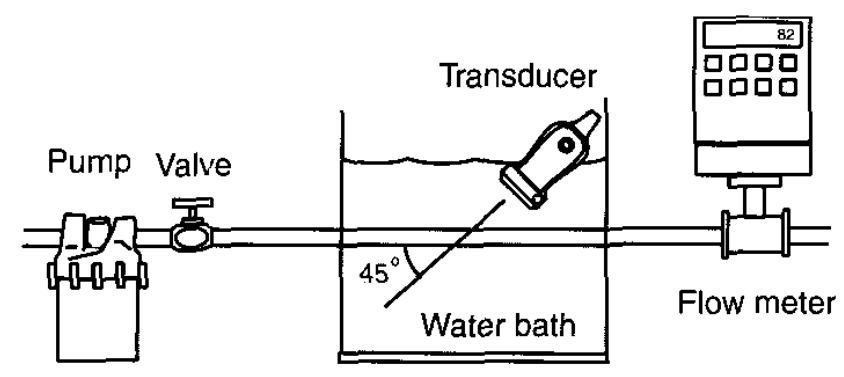

Fig. 8. Drawing of the flow rig for the parabolic flow experiment.

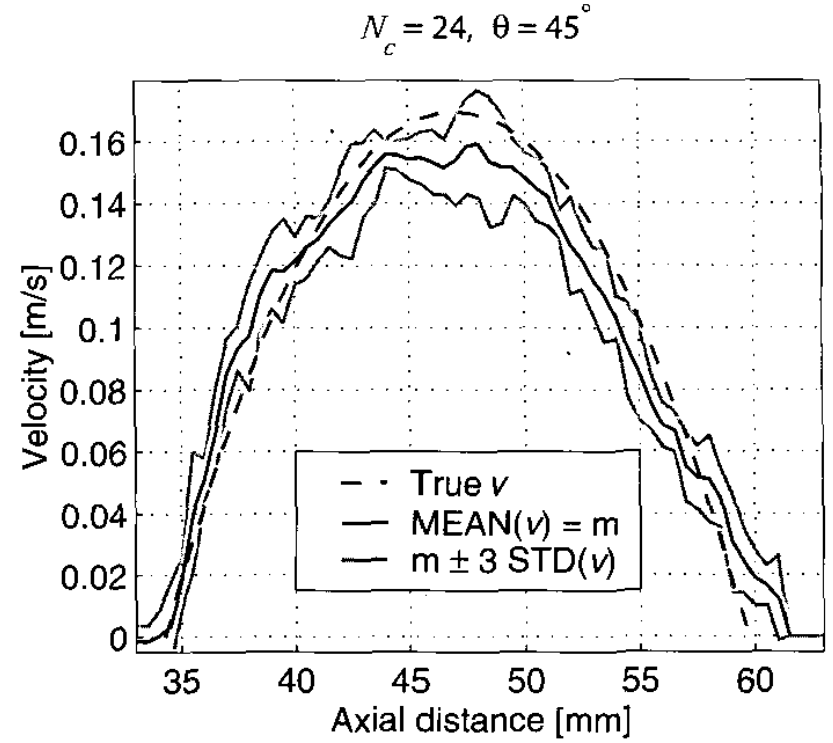

Fig. 9. Velocity profile measured from a flow phantom with a laminar, parabolic velocity profile. The average of 156 profiles are shown together with the true profile and the mean \pm 3 standard deviations.

mimicking fluid ("EU-DFS-BMF-ver. 1") (Dansk Fantom Service, Jyllinge, Denmark) was used. A 64-element linear array transducer with a nominal frequency of $7.5 \mathrm{MHz}$ and the parameters shown in Table I was used for the measurement. The angle between the acoustic axis of the transducer and the laminar flow was 45 degrees. Pulse emission and data acquisition were performed by a dedicated ultrasound system developed in our laboratory [27]. It can simultaneously emit arbitrary waveforms on 128 channels and can, in parallel, sample 64 channels at $40 \mathrm{MHz}$ and 12 bits.

The acquisition was done for 180 emissions using four groups of 17 elements [4], [6], which create spherical waves. The centers of these four groups were uniformly positioned across the 64-element active aperture. $N_{c}=24$ lines were used for each velocity estimate, and 156 velocity profiles were obtained. Each group of elements was set to combined emit a spherical wave to increase the signal-to-noise ratio. A linear frequency modulated pulse also was used in transmit to increase the transmitted energy, hence, the signalto-noise ratio. Compression of the received RF data was done with a modified, matched filter [24] prior to beamformation.

The results are shown in Fig. 9. The black solid line is the mean estimated velocity, the gray solid lines outline the velocity within \pm 3 standard deviations $(3 \sigma)$, and the black dashed line is the true ${ }^{2}$ velocity profile. The standard deviation varies between 1.7 and $6 \%$, with an average over the profile of $2.2 \%$ relative to the peak velocity of $0.17 \mathrm{~m} / \mathrm{s}$. The bias at the peak (at depth of $47.5 \mathrm{~mm}$ ) is $-11 \%$, with an average of $3.4 \%$ over the whole profile.

\footnotetext{
${ }^{2}$ This is the calculated velocity profile, based on the mass flow meter.
} 


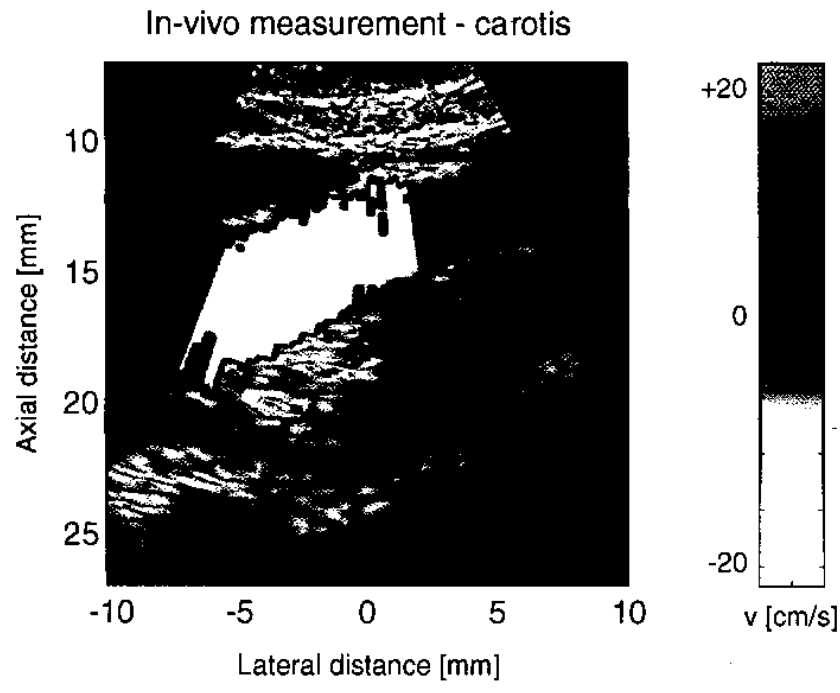

Fig. 10. In-vivo color flow image of the carotid artery scanned with a $7 \mathrm{MHz}$ linear array transducer on a 29 -year-old healthy male.

\section{In-Vivo Flow in Carotid Artery}

An in-vivo measurement was performed with the same measurement system setup and transducer as mentioned earlier. The carotid artery of a normal 29-year-old male was scanned, and the data was processed off line. The resulting image is shown in Fig. 10.

Both a B-mode, gray-scale image and a color flow map image were reconstructed from the same data. The high resolution images were first beamformed for each set of four emissions, and stationary echo canceling was done on the same set of high resolution images. Twenty-four pulseecho lines were used in the velocity estimation using cross correlation at a pulse repetition frequency of $7 \mathrm{kHz}$. Thus, more than 290 independent and full-size images can be acquired per second. The radial distance between two estimates is $0.5 \mathrm{~mm}$. The starting and ending angles of the color flow map are $\gamma \in\left[-21^{\circ}, 6^{\circ}\right]$, corresponding roughly to $\theta \in\left[47^{\circ}, 74^{\circ}\right]$, and the number of lines is 30 . The colorbar on the right indicates the velocity range from $-20 \mathrm{~cm} / \mathrm{s}$ (blue) to $+20 \mathrm{~cm} / \mathrm{s}$ (red). The discriminator for showing velocities was solely based on the velocity being over $0.01 \mathrm{~cm} / \mathrm{s}$, and no postprocessing was used on the estimates. It can be seen that the flow is confined to lie within the carotid artery. The displayed color varies as a function of angle, and this is due to the $\cos (\theta)$ factor in (5). Thus, this image demonstrates that synthetic aperture flow imaging can be done in-vivo.

\section{Summary}

The new synthetic aperture acquisition approach has the advantage that data is acquired simultaneously from all directions of the image. Therefore, it is possible to follow moving objects continuously, which is of considerable benefit in velocity imaging. The flow image can be calcu- lated continuously for all positions at the same time, and the frame rate can be maintained at any rate up to the pulse repetition frequency, which is in the kilohertz range. Having continuous data also makes it possible to have any kind of filter for the separation between slow moving tissue and the blood because data is continuous and no initialization of the filter is necessary. Any filtering can then be done, and very slow moving flow can be separated from moving tissue. The estimators for the flow also can work on any number of samples limited only by the time over which the flow can be assumed stationary. Often 100 to 200 pulse echo lines can be used in the estimation of the flow with this method, and potentially that should reduce the variance by a factor of 10 to 20 compared to conventional flow imaging. The continuous data acquisition also makes it possible to have any number of velocity spectra calculated simultaneously, albeit with a lower effective pulse repetition frequency. Thus, the velocity distribution before and after a stenosis can be studied by simultaneously displaying two velocity spectra. Using SA data acquisition also opens up the possibility for arbitrary focusing methods. This can be beneficial in transverse flow imaging, in which either a transverse oscillating field is synthesized [28] or a signal following the flow is sought [29]. The last method also can reduce the decorrelation of the data over several pulse acquisitions and thereby can further improve the standard deviation of the flow estimates.

The application of SA acquisition also improves the image quality, as it makes it possible to have focusing in both transmit and receive. The signal-to-noise ratio can reach acceptable levels by using codes and a set of elements in transmit. Thus, the suggested method makes it possible to devise a complete ultrasound SA system with both anatomic and flow-imaging capabilities.

\section{ACKNOWLEDGMENT}

The authors would like to thank Rasmus T. Bjerngaard and Kjeld Martinsen for setting up the flow rig. This work was supported by grant 9700883, 9700563, and 26-01-0178 from the Danish Science Foundation and by B-K Medical A/S, Denmark.

\section{REFERENCES}

[1] L. F. Nock and G. E. Trahey, "Synthetic receive aperture imaging with phase correction for motion and for tissue inhomogeneities-Part I: Basic principles," IEEE Trans. Ultrason., Ferroelect., Freq. Contr., vol. 39, pp. 489-495, 1992.

[2] G. E. Trahey and L. F. Nock, "Synthetic receive aperture imaging with phase correction for motion and for tissue inhomogeneities-Part II: Effects of and correction for motion," IEEE Trans. Ultrason., Ferroelect., Freq. Contr., vol. 39, pp. 496-501, 1992.

[3] J. T. Ylitalo, "On the signal-to-noise ratio of a synthetic aperture ultrasound imaging method," Eur. J. Ultrasound, vol. 3, no. 3, pp. $277-281,1996$.

[4] M. Karaman, P. C. Li, and M. O'Donnell, "Synthetic apertüre imaging for small scale systems," IEEE Trans. Ultrason., Ferroelect., Freq. 'Contr., vol. 42, pp. 429-442, 1995. 
[5] G. R. Lockwood, J. R. Talman, and S. S. Brunke, "Real-tine 3-D ultrasound imaging using sparse synthetic aperture beamforming:" IEEE Trans. Ultrason., Ferroelect., Freq. Contr., vol. 45, pp. 980-988, 1998.

[6] M. O'Donnell and L. J. Thomas, "Efficient synthetic aperture imaging from a circular aperture with possible application to catheter-based imaging," IEEE Trans, Ultrason., Ferroelect., Freq. Contr., vol. 39, pp. 366-380, 1992.

[7] C. Passmann and H. Ermert, "Adaptive $150 \mathrm{MHz}$ ultrasound imaging of the skin and the eye using an optimal combination of short pulse mode and pulse compression mode," in Proc. IEEE Ultrason. Symp. 1995, pp. 129l-1294.

[8] R. Y. Chiao, L. J. Thomas, and S. D. Silverstein, "Sparse array imaging with spatially-encoded transmits," in Proc. IEEE Ultrason. Symp., 1997, pp. 1679-1682.

[9] T. Misaridis, "Ultrasound imaging using coded signals," Ph.D. dissertation, Ørsted•DTU, Technical Univ. Denmark, Lyngby, Denmark, 2001.

[10] S. I. Nikolov, "Synthetic aperture tissue and flow ultrasound imaging," Ph.D. dissertation, Ørsted•DTU, Technical Univ. Denmark, Lyngby, Denmark, 2001.

[11] H. S. Bilge, M. Karaman, and M. O'Donnell, "Motion estimation using common spatial frequencies in synthetic aperture imaging," in Proc. IEEE Ultrason. Symp., 1996, pp. 1551-1554.

[12] M. Karaman, H. S. Bilge, and M. O'Donnell, "Adaptive multielement synthetic aperture imaging with motion and phase aberation correction," IEEE Trans. Ultrason., Ferroelect., Freq. Contr., vol. 42, pp. 1077-1087, 1998.

[13] S. I. Nikolov, K. Gammelmark, and J. A. Jensen, "Recursive ultrasound imaging;" in Proc. IEEE Ultrason. Symp., 1999, pp. $1621-1625$.

[14] C. Kasai, K. Namekawa, A. Koyano, and R. Omoto, "Real-time two-dimensional blood flow imaging using an autocorrelation technique," IEEE Trans. Sonics Ultrason., vol. 32, pp. 458-463, 1985

[15] O. Bonnefous and P. Pesqué, "Time domain formulation of pulse-Doppler ultrasound and blood velocity estimation by cross correlation," Ultrason. Imag., vol. 8, pp. 73-85, 1986.

[16] S. G. Foster, "A pulsed ultrasonic flowmeter employing time domain methods," Ph.D. dissertation, Department Elec. Eng., Univ. Illinois, Urbana, 1985.

[17] P. M. Embree, "The accurate ultrasonic measurement of the volume flow of blood by time domain correlation," Ph.D. dissertation, Department Elec. Eng., Univ. Illinois, Urbana, 1986.

[18] S. G. Foster, P. M. Embree, and W. D. O'Brien, "Flow velocity profile via time-domain correlation: Error analysis and computer simulation," IEEE Trans. Ultrason., Femolect., Freq. Contr., vol. 37, pp. 164-175, 1990.

[19] J. A. Jensen, Estimation of Blood Velocities Using Ultrasound: A Signal Processing Approach. New York: Cambridge Univ. Press, 1996.

[20] S. I. Nikolıv, K. Gammelmark, and J. A. Jensen, "Velocity estimation using recursive ultrasound imaging and spatially encoded signals," in Proc. IEEE Vltrason. Symp., 2000, pp. 1473-1477.

[21] S. I. Nikolov and J. A. Jensen, "Velocity estimation using synthetic aperture imaging;" in Proc. IEEE Ultrason. Symp., 2001, pp. 1409-1412.

[22] K. L. Gammelmark and J. A. Jensen, "Multi-element synthetic transmit aperture imaging using temporal encoding," in Proc. SPIE: Progress in Biomedical Optics and Imaging, vol. 3, pp. 25-36, 2002.

[23] C. R. Hazard and G. R. Lockwood, "Effects of motion artifacts on a synthetic aperture beamformer for real-time $3 D$ ultrasound," in Proc. IEEE Ultrason. Symp., 1999, pp. 1221-1224.
[24] T. X. Misaridis and J. A. Jensen, "An effective coded excitation scheme based on a predistorted FM signal and an optimized digital filter," in Proc. IEEE Ultrason. Symp., 1999, pp. 15891593.

[25] R. B. Dilley and A. Fronek, "Quantitative velocity measurements in arterial disease of the lower extremity," in Non-Invasive Diagnostic Techniques in Vascular Disease. E. F. Bernstein, Ed. St. Louis, MO: Mosby, 1979, pp. 294-303.

[26] J. A. Jensen, "Field: A program for simulating ultrasound systems," Med. Biol. Eng. Comp., vol. 4, Suppl. 1, pt. 1, pp. 351$353,1996 \mathrm{~b}$.

[27] J. A. Jensen, O. Holm, L. J. Jensen, H. Bendsen, H. M. Pedersen, K. Salomonsen, J. Hansen, and S. Nikolov, "Experimental ultrasound system for real-time synthetic imaging," in Proc. IEEE Ultrason. Symp., 1999, pp. 1595-1599.

[28] J. A. Jensen and P. Munk, "An improved estimation and focusing scheme for vector velocity estimation," in Proc. IEEE Ultrason. Symp., 1999, pp. 1465-1470.

[29] J. A. Jensen and I. R. Lacasa, "Estimation of blood velocity vectors using transverse ultrasound beam focusing and crosscorrelation," in Proc. IEEE Ultrason. Symp., 1999, pp. 14931497.

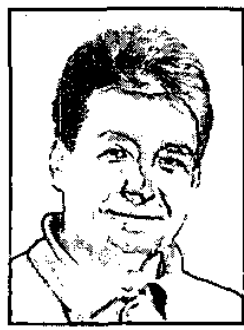

Svetoslav Ivanov Nikolov received the M.Sc. degree in electrical engineering and M.B.A. degree in international business relations from the Technical University of Sofia Sofia, Bulgaria, in 1996 and 1997, respectively. In 2001 he received a Ph.D. degree from the Technical University of Denmark (DTU), Lyngby. His dissertation explored approaches for synthetic aperture tissue and flow imaging, and the possibilities for real-time 3-D imaging.

After completing his doctoral work, he stayed on at DTU as an assistant professor. He teaches digital design and programming. His research interests include signal and image processing and their application to ultrasound imaging.

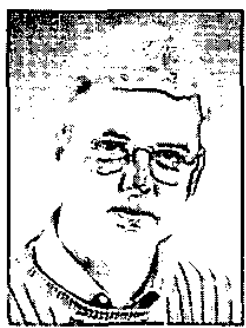

Jørgen Arendt Jensen (M'93-S'02) earned his M.S. degree in electrical engineering in 1985 and the Ph.D. degree in 1989 , both from the Technical University of Denmark (DTU), Lyngby, Denmark. He received the Dr.Techn. degree from the university in 1996. He has published a number of papers on signal processing and medical ultrasound and the book Estimation of Blood Velocities Using Ultrasound (Cambridge Univ. Press) in 1996. He has been a visiting scientist at Duke University, Durham, NC, Stanford University, Palo Alto, CA, and the University of Illinois at Urbana-Champaign. He is currently a full professor of Biomedical Signal Processing at the DTU in the Department of Information Technology and head of the Center for Fast Ultrasound Imaging. He has given courses on blood velocity estimation at both Duke University and University of Illinois, and he teaches biomedical signal processing and medical imaging at the DTU. He arranged an international summer school on advanced ultrasound imaging in 1999. 